\title{
Discrete-Time Multioverlapping Controller Design for Structural Vibration Control of Tall Buildings under Seismic Excitation
}

\author{
Francisco Palacios-Quiñonero, ${ }^{1}$ Josep Rubió-Massegú, ${ }^{1}$ \\ Josep M. Rossell, ${ }^{1}$ and Hamid Reza Karimi ${ }^{2}$ \\ ${ }^{1}$ Departament de Matemàtica Aplicada III, Universitat Politècnica de Catalunya (UPC), \\ Avinguda Bases de Manresa 61-73, 08242 Manresa, Barcelona, Spain \\ ${ }^{2}$ Department of Engineering, Faculty of Engineering and Science, University of Agder (UiA), \\ 4898 Grimstad, Norway \\ Correspondence should be addressed to Francisco Palacios-Quiñonero, \\ francisco.palacios@upc.edu
}

Received 22 June 2012; Revised 9 September 2012; Accepted 10 September 2012

Academic Editor: Jun $\mathrm{Hu}$

Copyright (c) 2012 Francisco Palacios-Quiñonero et al. This is an open access article distributed under the Creative Commons Attribution License, which permits unrestricted use, distribution, and reproduction in any medium, provided the original work is properly cited.

In this paper, a computationally effective strategy to obtain multioverlapping controllers via the Inclusion Principle is applied to design discrete-time state-feedback multioverlapping LQR controllers for seismic protection of tall buildings. To compute the corresponding control actions, the proposed semidecentralized controllers only require state information from neighboring stories. This particular configuration of information exchange allows introducing a dramatic reduction in the transmission range required for a wireless implementation of the communication system. To investigate the behavior of the proposed semidecentralized multioverlapping controllers, a proper simulation model has been designed. This model includes semiactive actuation devices with limited force capacity, control sampling times consistent with the communication latency, time-delayed state information, and communication failures. The performance of the proposed multioverlapping controllers has been assessed through numerical simulations of the seismic response of a 20 -story building with positive results.

\section{Introduction}

Over the last decades, problems of ever increasing complexity have been considered in the field of Structural Vibration Control (SVC). Current SVC systems for seismic protection of tall buildings can involve a large number of sensors and actuation devices and a wide and sophisticated communication network [1-3]. Semidecentralized control strategies, which can operate using only state information from neighboring stories, are especially relevant for 
wireless implementations of the communication system. Semidecentralized state-feedback LQR controllers were proposed by Wang and Lynch in [4], and the study was extended to state-feedback $H_{\infty}$ controllers by Wang et al. in [5]. The numerical and experimental results obtained in these works clearly indicate that the proposed semidecentralized control strategies are specially suitable for SVC of tall buildings with wireless communications. It should be highlighted, however, that important computational difficulties can arise when applying these control design strategies to large buildings. The LQR controller design presented in [4] uses a variant of the heuristic iterative procedure proposed by Lunze in [6], and the $H_{\infty}$ controller design in [5] is based on a Linear Matrix Inequality formulation (LMI). For large-dimensional problems, a great computational effort is required by the iterative procedure used in the LQR design. Analogously, solving large-dimensional convex optimization problems with LMI constraints is also a costly computational task.

In this context, the design of semidecentralized controllers using multioverlapping decompositions based on the Inclusion Principle (IP) is a very interesting option [7-12]. Broadly speaking, the IP allows decomposing the original large-dimensional problem into a set of low-dimensional decoupled problems. This decomposition takes advantage of the particular structure of the original system and can help to significantly reduce the computational effort. Examples of successful applications of the IP to SVC can be found in [13-15]. Recently, an effective computational strategy to design semidecentralized multioverlapping controllers based on a sequential application of the IP was presented by Palacios-Quiñonero et al. in [16]. In that work, semidecentralized multioverlapping LQR controllers are designed for seismic protection of a four-story building with positive results. However, it has to be noted that all these applications of the IP to SVC have been conducted using small buildings, continuous-time models, and assuming highly idealized conditions, such as active force actuators with unrestricted force capacity and communication systems with no failures nor delays.

The main contribution of the present paper is to present a large-scale application of the IP to the design of semidecentralized controllers for SVC, paying special attention to some aspects of practical relevance. More specifically, the computational strategy proposed in [16] is applied to design discrete-time state-feedback multioverlapping LQR controllers to mitigate the seismic response of a 20-story building. Moreover, to gain a meaningful insight into the behavior of the proposed multioverlapping controllers, the models used in the numerical simulations include some factors of practical relevance such as control sampling rates, realistic implementation of the control actions, time-delayed state information, and communication latency and failures. One of the main difficulties encountered when applying the IP to discrete-time controller design is that the natural structure of the continuous-time model is lost in the discretization process. To overcome this difficulty, the discretization process has been carried out on the expanded decoupled subsystems. The results obtained in the numerical simulations confirm the excellent characteristics of the proposed semidecentralized multioverlapping controllers for SVC of large buildings.

The organization of the paper is as follows: In Section 2, a detailed derivation of the continuous-time state-space model for an $n$-story building is presented. Section 3 begins with a summary discussion on the design of discrete-time state-feedback centralized LQR controllers. Next, the main ideas involved in the design of discrete-time state-feedback multioverlapping LQR controllers are briefly presented. In Section 4, three mathematical models used to conduct the numerical simulations of the building seismic response are presented: (i) Basic building model, which consists in a discrete-time approximation of the continuous-time state-space model with a small sampling time and no control action. 


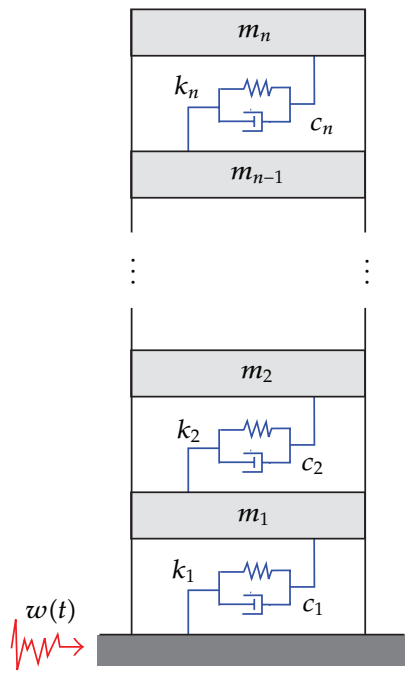

Figure 1: Building lumped-mass model.

(ii) Centralized control model, which implements the discrete-time centralized LQR controller with perfect state knowledge, small sampling time, and ideal semiactive force actuators with limited force capacity. (iii) Multioverlapping control model. This case implements a discretetime multioverlapping controller considering semiactive actuation devices with limited force capacity, a control sampling time consistent with the communication latency, time-delayed state information, and communication failures. Finally, in Section 5, the control design methodology presented in Section 3 and the simulation models introduced in Section 4 are applied to a particular 20-story building to assess the performance of the proposed multioverlapping controllers.

\section{Continuous-Time Building Model}

Let us consider the $n$-story building schematically displayed in Figure 1, which is modeled as a lumped-mass planar system with displacements in the direction of the ground motion. The building motion can be described by the second-order differential equation:

$$
M \ddot{q}(t)+C \dot{q}(t)+K q(t)=T_{u} u(t)+T_{\omega} \omega(t),
$$

where

$$
q(t)=\left[q_{1}(t), \ldots, q_{n}(t)\right]^{T}
$$

is the vector of story displacements with respect to the ground, and $q_{i}(t)$ represents the displacement of the ith story. $M, C, K$ are the mass, damping, and stiffness matrices, respectively. The vector of control actions is

$$
u(t)=\left[u_{1}(t), \ldots, u_{n}(t)\right]^{T}
$$




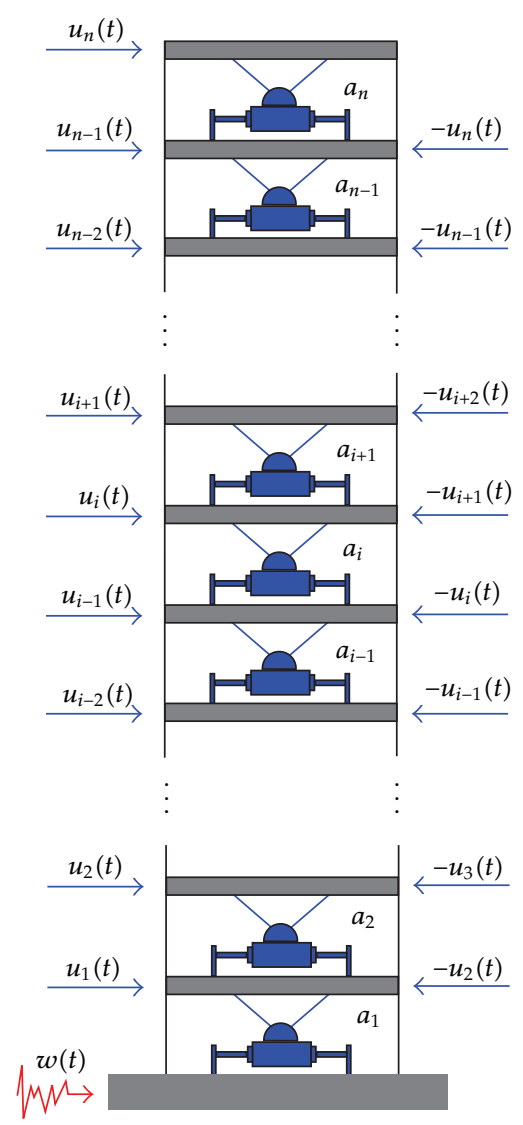

Figure 2: Actuation scheme.

where $u_{i}(t)$ represents the control force exerted by the actuation device $a_{i}$ (see Figure 2), and $T_{u}$ is the control location matrix. The seismic ground acceleration is $\omega(t)$, and $T_{\omega}$ denotes the disturbance input matrix. The mass and stiffness matrices have the following structures:

$$
\begin{gathered}
M=\left[\begin{array}{llll}
m_{1} & & & \\
& \cdots & & \\
& & \cdots & \\
& & & m_{n}
\end{array}\right], \\
K=\left[\begin{array}{cccccc}
k_{1}+k_{2} & -k_{2} & & & \\
-k_{2} & k_{2}+k_{3} & -k_{3} & & & \\
& \cdots & \cdots & \cdots & & \\
& & \cdots & \cdots & \cdots & \\
& & & -k_{n-1} & k_{n-1}+k_{n} & -k_{n} \\
& & & & -k_{n} & k_{n}
\end{array}\right],
\end{gathered}
$$

where $m_{i}$ and $k_{i}$ represent, respectively, the mass and stiffness of the $i$ th story. When the values of the story damping coefficients $c_{i}, 1 \leq i \leq n$, are known, a damping matrix $C$ with 
the same structure as $K$ can be obtained by replacing $k_{i}$ by $c_{i}$ in (2.5). Alternatively, a tridiagonal damping matrix in the form

$$
C=\alpha_{0} M+\alpha_{1} K
$$

can be computed following the Rayleigh damping approach by setting the damping ratio values for two selected natural frequencies [17]. For the actuation system schematically depicted in Figure 2, the control location matrix has dimensions $n \times n$ and the following structure:

$$
T_{u}= \begin{cases}{\left[T_{u}\right]_{i, i}=1,} & \text { for } 1 \leq i \leq n, \\ {\left[T_{u}\right]_{i, i+1}=-1,} & \text { for } 1 \leq i<n \\ {\left[T_{u}\right]_{i, j}=0,} & \text { otherwise }\end{cases}
$$

where $\left[T_{u}\right]_{i, j}$ denotes the element in the $i$ th row and $j$ th column of $T_{u}$. Finally, the disturbance input matrix is

$$
T_{w}=-M[1]_{n \times 1}
$$

where $[1]_{n \times 1}$ is a column vector of dimension $n$ with all its entries equal to 1 .

Now, we take the state vector

$$
x_{I}(t)=\left[\begin{array}{l}
q(t) \\
\dot{q}(t)
\end{array}\right]
$$

and derive a first-order state-space model

$$
S_{I}: \dot{x}_{I}(t)=A_{I} x_{I}(t)+B_{I} u(t)+E_{I} \omega(t) .
$$

The state, control, and disturbance input matrices are, respectively,

$$
A_{I}=\left[\begin{array}{cc}
{[0]_{n \times n}} & I_{n} \\
-M^{-1} K & -M^{-1} C
\end{array}\right], \quad B_{I}=\left[\begin{array}{c}
{[0]_{n \times n}} \\
M^{-1} T_{u}
\end{array}\right], \quad E_{I}=\left[\begin{array}{c}
{[0]_{n \times 1}} \\
-[1]_{n \times 1}
\end{array}\right],
$$

where $[0]_{r \times s}$ represents a zero matrix of dimensions $r \times s$, and $I_{n}$ is the identity matrix of dimension $n$. Next, we consider a new state vector

$$
x(t)=\left[x_{1}(t), \ldots, x_{2 n}(t)\right]^{T},
$$


which groups together the interstory drifts and interstory velocities in increasing order

$$
x(t)= \begin{cases}x_{1}(t)=q_{1}(t), & \\ x_{2}(t)=\dot{q}_{1}(t), & \\ x_{2 i-1}(t)=q_{i}(t)-q_{i-1}(t), & \text { for } 1<i \leq n, \\ x_{2 i}(t)=\dot{q}_{i}(t)-\dot{q}_{i-1}(t), & \text { for } 1<i \leq n\end{cases}
$$

Using the change of basis

$$
x(t)=P x_{I}(t)
$$

defined by the $2 n \times 2 n$ matrix

$$
P= \begin{cases}p_{1,1}=1, p_{2, n+1}=1, & \\ p_{2 i-1, i-1}=-1, p_{2 i-1, i}=1, & \text { for } 1<i \leq n, \\ p_{2 i, n+i-1}=-1, p_{2 i, n+i}=1, & \text { for } 1<i \leq n, \\ p_{i, j}=0, & \text { otherwise }\end{cases}
$$

we obtain the new state space model:

$$
S: \dot{x}(t)=A x(t)+B u(t)+E \omega(t)
$$

where

$$
A=P A_{I} P^{-1}, \quad B=P B_{I}, \quad E=P E_{I} .
$$

In this work, we restrict our attention to the interstory drifts as output variables. The output vector can then be obtained as

$$
y(t)=\left[y_{1}(t), \ldots, y_{n}(t)\right]^{T}=C_{y} x(t),
$$

where $C_{y}$ is a matrix of dimensions $n \times 2 n$ with the following structure:

$$
C_{y}= \begin{cases}{\left[C_{y}\right]_{i, 2 i-1}=1,} & \text { for } 1 \leq i \leq n \\ {\left[C_{y}\right]_{i, j}=0,} & \text { otherwise. }\end{cases}
$$




\section{Control Design}

To design a discrete-time centralized state-feedback LQR controller for the $n$-story building model presented in the previous section, we begin by considering the continuous-time system:

$$
S_{c}: \dot{x}(t)=A x(t)+B u(t)
$$

obtained from (2.16) by removing the disturbance term $E \omega(t)$. The discrete-time system corresponding to the zero-hold approximation of (3.1) with sampling time $\tau$ is

$$
\left\{S_{c}\right\}_{\tau}: x[k+1]=A_{\tau} x[k]+B_{\tau} u[k]
$$

where

$$
A_{\tau}=e^{A \tau}, \quad B_{\tau}=\int_{0}^{\tau} e^{A t} B d t
$$

Next, we consider the discrete-time state-feedback controller:

$$
u[k]=-G_{\tau} x[k]
$$

and the quadratic index:

$$
J(x, u)=\sum_{k=0}^{k=\infty} x[k]^{T} Q x[k]+u[k]^{T} R u[k]
$$

where $Q$ is a symmetric positive semidefinite matrix, and $R$ is a symmetric positive definite matrix. The control gain matrix $G_{\tau}$ that minimizes (3.5) under constraints (3.2) and (3.4) can be computed as

$$
G_{\tau}=\left(R+B_{\tau}^{T} P B_{\tau}\right)^{-1} B_{\tau}^{T} P A_{\tau}
$$

where $P$ is the solution of the discrete-time Riccati equation:

$$
A_{\tau}^{T} P A_{\tau}-P+Q-A_{\tau}^{T} P B_{\tau}\left(R+B_{\tau}^{T} P B_{\tau}\right)^{-1} B_{\tau}^{T} P A_{\tau}=0
$$

To design a multioverlapping controller that is able to compute the control actions $u_{i}[k]$ using only state information corresponding to neighboring stories, we consider the $n$ story building decomposed into a sequence of $n-1$ two-story overlapped subsystems

$$
S^{(i)}=\left[s_{i}, s_{i+1}\right], \quad 1 \leq i \leq n-1,
$$




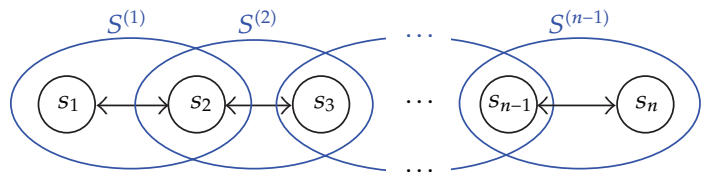

Figure 3: Decomposition in two-story overlapping subsystems.

where $s_{i}$ represents the $i$ th story. This overlapping decomposition is schematically depicted in Figure 3. Following the sequential multioverlapping decomposition strategy proposed in [16], the initial continuous-time system (3.1) can be conveniently expanded to form a new continuous-time system:

$$
\tilde{S}: \dot{\tilde{x}}(t)=\tilde{A} \tilde{x}(t)+\tilde{B} \tilde{u}(t)
$$

where the state matrix $\widetilde{A}$ and the control input matrix $\widetilde{B}$ are block diagonal. The expanded system $\widetilde{S}$ can then be decomposed into a sequence of decoupled continuous-time subsystems:

$$
\widetilde{S}^{(i)}: \dot{\tilde{x}}^{(i)}(t)=\widetilde{A}^{(i)} \widetilde{x}^{(i)}(t)+\widetilde{B}^{(i)} \widetilde{u}^{(i)}(t), \quad 1 \leq i \leq n-1
$$

For the continuous-time subsystems $\widetilde{S}^{(i)}$, we compute discrete-time zero-hold approximations with sampling time $\widehat{\tau}$ :

$$
\left\{\widetilde{S}^{(i)}\right\}_{\widehat{\tau}}: \tilde{x}^{(i)}[k+1]=\widetilde{A}_{\widehat{\tau}}^{(i)} \tilde{x}^{(i)}[k]+\widetilde{B}_{\widehat{\tau}}^{(i)} \widetilde{u}^{(i)}[k], \quad 1 \leq i \leq n-1,
$$

where

$$
\widetilde{A}_{\widehat{\tau}}^{(i)}=e^{\widetilde{A}^{(i)} \widehat{\tau}}, \quad \widetilde{B}_{\widehat{\tau}}^{(i)}=\int_{0}^{\widehat{\tau}} e^{\widetilde{A}^{(i)} t} \widetilde{B}^{(i)} d t
$$

and consider the local quadratic indexes:

$$
\widetilde{J}^{(i)}\left(\tilde{x}^{(i)}, \tilde{u}^{(i)}\right)=\sum_{k=0}^{k=\infty}\left\{\tilde{x}^{(i)}[k]\right\}^{T} Q^{(i)} \tilde{x}^{(i)}[k]+\left\{\tilde{u}^{(i)}[k]\right\}^{T} R^{(i)} \tilde{u}^{(i)}[k], \quad 1 \leq i \leq n-1,
$$

to compute local discrete-time LQR controllers

$$
\tilde{u}^{(i)}[k]=-\widetilde{G}_{\widehat{\tau}}^{(i)} \tilde{x}^{(i)}[k], \quad 1 \leq i \leq n-1,
$$


which minimize the indexes (3.13) under constraints (3.11) and (3.14). Finally, the sequence of expanded local control matrices $\widetilde{G}_{\widehat{\tau}}^{(i)}$ is contracted back to a control gain matrix $\widehat{G}_{\widehat{\tau}}$ in order to define a discrete-time multioverlapping controller:

$$
\widehat{u}[k]=-\widehat{G}_{\widehat{\tau}} x[k]
$$

for the original discrete-time system (3.2).

Remark 3.1. The expansion-contraction procedure associated to the design of multioverlapping controllers for large buildings is only outlined in this section. For clarity and simplicity, a detailed account of this procedure has not been included in the paper. However, a complete presentation of this background material together with some practical applications to SVC of small buildings can be found in $[15,16]$.

Remark 3.2. The expanded block-diagonal system (3.9) can only be computed when the matrices of the initial state-space system have a suitable zero-nonzero block structure. For the building model (2.1), the initial state-space system (3.1) has a proper structure. However, this structure is lost in the discretization process and the expansion-decoupling process can no longer be applied to the discrete-time state-space system (3.2). To overcome this difficulty, the expansion-decoupling process is first completed for the continuous-time system and, after that, the discretization process is carried out on the continuous-time expanded decoupled subsystems (3.10) to obtain the discrete-time expanded decoupled subsystems (3.11).

Remark 3.3. It should be noted that the control gain matrix $G_{\tau}=\left[\left(g_{\tau}\right)_{i, j}\right]$ given in (3.6) is a full matrix of size $n \times 2 n$, and the full state is required to compute the control action for the actuation device $a_{i}$ :

$$
u_{i}[k]=\sum_{j=1}^{j=2 n}\left(g_{\tau}\right)_{i, j} x_{j}[k], \quad 1 \leq i \leq n .
$$

In contrast, the multioverlapping control matrix $\widehat{G}_{\hat{\tau}}=\left[\left(\widehat{g}_{\hat{\tau}}\right)_{i, j}\right]$ has a block-tridiagonal structure and only requires a reduced number of 4-6 states to compute the control action for each actuation device. More specifically, we have

$$
\widehat{u}[k]=\left\{\begin{array}{l}
\widehat{u}_{1}[k]=\sum_{j=1}^{j=4}\left(\widehat{g}_{\widehat{\tau}}\right)_{1, j} x_{j}[k], \\
\widehat{u}_{i}[k]=\sum_{j=2 i-3}^{j=2 i+2}\left(\widehat{g}_{\widehat{\tau}}\right)_{i, j} x_{j}[k], \quad \text { for } 1<i<n, \\
\widehat{u}_{n}[k]=\sum_{j=2 n-3}^{j=2 n}\left(\widehat{g}_{\widehat{\tau}}\right)_{n, j} x_{j}[k] .
\end{array}\right.
$$

Remark 3.4. For clarity and simplicity, the controllers presented in this section have been computed following an LQR approach. However, it has to be highlighted that other control strategies are also possible. For example, an application of the IP to the design of semidecentralized static output-feedback controllers for SVC can be found in [14]. 


\section{Simulation Models}

One of the main objectives of the present work is to gain a meaningful insight into the behavior of semidecentralized multioverlapping controllers through numerical simulations. To this end, the simulation models have to include some relevant factors such as sampling rates, realistic implementation of the control actions, time-delayed state information, and communication latency and failures. Trying to achieve a proper balance between simplicity and accuracy, we have considered a simulation framework formed by three different models: (i) Basic building model, which consists in a discrete-time approximation of the continuoustime state-space model (3.1) with a small basic sampling time $\tau$ and no control action. (ii) Centralized control model, which implements the discrete-time LQR controller given in (3.4) with perfect state knowledge and the basic sampling time $\tau$. The actuation devices, however, are assumed to be ideal semiactive force actuators with limited force capacity. (iii) Multioverlapping control model. This case implements the discrete-time multioverlapping controller given in (3.15) considering semiactive actuation devices with limited force capacity, a control sampling time $\widehat{\tau}>\tau$ consistent with the communication latency, time-delayed state information, and communication failures. In all the cases, the interstory drifts are taken as output variables.

The basic building model is:

$$
\mathcal{M}_{\tau}:\left\{\begin{array}{l}
x[k+1]=A_{\tau} x[k]+E_{\tau} \omega_{\tau}[k] \\
y[k]=C_{y} x[k]
\end{array}\right.
$$

where $A_{\tau}$ is the discrete-time state matrix in (3.3); $E_{\tau}$ is the discrete-time disturbance input matrix, which can be computed as

$$
E_{\tau}=\int_{0}^{\tau} e^{A t} E d t
$$

with $A$ and $E$ representing, respectively, the state and disturbance input continuous-time matrices; and $\omega_{\tau}[k]=\omega(k \tau)$ is the sampled disturbance. The vector of interstory drifts is computed with the output matrix $C_{y}$ given in (2.19). A good approximation of the uncontrolled seismic response of the building can be obtained using the basic building model $\boldsymbol{M}_{\tau}$ with a small sampling time $\tau$.

The centralized control model is:

$$
\overline{\mathcal{M}}_{\tau}:\left\{\begin{array}{l}
u[k]=-G_{\tau} x[k], \\
x[k+1]=A_{\tau} x[k]+B_{\tau} \sigma(u[k])+E_{\tau} \omega_{\tau}[k], \\
y[k]=C_{y} x[k],
\end{array}\right.
$$

where the sampling time $\tau$, the matrices $A_{\tau}, E_{\tau}$, and $C_{y}$, and the sampled disturbance $\omega_{\tau}[k]$ are the same as those used in (4.1); and $B_{\tau}$ is the discrete-time input-control matrix defined in (3.3). The vector of control actions

$$
u[k]=\left[u_{1}[k], \ldots, u_{n}[k]\right]^{T},
$$




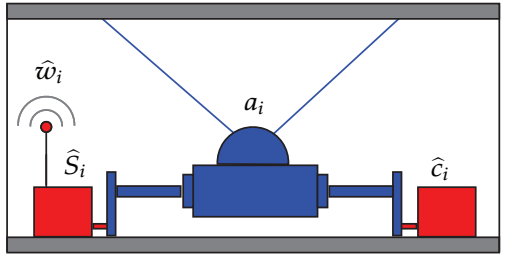

Figure 4: Actuation-communication system for the multioverlapping control model.

is computed using the discrete-time centralized control gain matrix $G_{\tau}$ given in (3.6), and the vector of control forces is

$$
\sigma(u[k])=\left[\sigma\left(u_{1}[k]\right), \ldots, \sigma\left(u_{n}[k]\right)\right]^{T} .
$$

In this section, the actuation devices $a_{i}$ are modeled as ideal semiactive force actuators with maximum actuation force $\left[f_{\max }\right]_{i}$. For a given control action $u_{i}[k]$, the actual control force exerted by the actuation device $a_{i}$ is

$$
\sigma\left(u_{i}[k]\right)= \begin{cases}u_{i}[k] & \text { if } u_{i}[k] \cdot v_{i}[k]<0, \text { and }\left|u_{i}[k]\right| \leq\left[f_{\max }\right]_{i} \\ \operatorname{sgn}\left(u_{i}[k]\right) \cdot\left[f_{\max }\right]_{i} & \text { if } u_{i}[k] \cdot v_{i}[k]<0, \text { and }\left|u_{i}[k]\right|>\left[f_{\max }\right]_{i} \\ 0 & \text { if } u_{i}[k] \cdot v_{i}[k] \geq 0,\end{cases}
$$

where $v_{i}[k]$ is the corresponding interstory velocity, and $\operatorname{sgn}(x)=x /|x|$ is the signum function. In the centralized control model, we assume an ideal communication system, which can provide a perfect knowledge of the full state vector $x[k]$. This model is used as a reference in the performance assessment of the multioverlapping controller.

In the multioverlapping control model, we consider the actuation-communication system schematically depicted in Figure 4 , consisting in an actuation device $a_{i}$, a sensor unit $\widehat{s}_{i}$, a local control unit $\widehat{c}_{i}$, and a wireless communication unit $\widehat{w}_{i}$. The actuation device $a_{i}$ produces the semiactive implementation of the control actions defined in (4.6). The sensor unit $\widehat{s}_{i}$ is an ideal sensor that provides an exact measurement of the local state

$$
\widehat{x}_{i}[k]=\left[\begin{array}{l}
y_{i}[k] \\
v_{i}[k]
\end{array}\right]
$$

where $y_{i}[k]$ and $v_{i}[k]$ denote the local interstory drift and velocity, respectively. To model the operation of the local control unit, we introduce the controller sampling tim

$$
\widehat{\tau}=\widehat{r} \tau, \quad \widehat{r}>1,
$$

where $\tau$ is the basic sampling time used in (4.1) and (4.3), and $\widehat{r}$ can be understood as the maximum number of sampling steps that can be spent by $\widehat{c}_{i}$ to collect state information from 


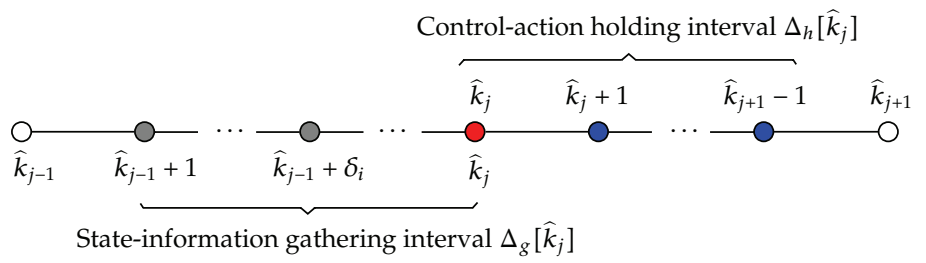

Figure 5: Time intervals for state information gathering and control action holding.

neighboring stories through the communication unit $\widehat{w}_{i}$. We also consider the update-control times

$$
\widehat{k}_{j}=j \widehat{r}, \quad j \geq 1,
$$

and define the interval of state-information gathering

$$
\Delta_{g}\left[\widehat{k}_{j}\right]=\left[\widehat{k}_{j-1}+1, \ldots, \widehat{k}_{j}\right]
$$

and the interval of control-action holding

$$
\Delta_{h}\left[\widehat{k}_{j}\right]=\left[\widehat{k}_{j}, \ldots, \widehat{k}_{j+1}-1\right]
$$

which are schematically represented in Figure 5 . The operation of the local control unit $\widehat{c}_{i}$ has been modeled in accordance with the following set of basic principles:

(P.1) The local control action $\widehat{u}_{i}[k]$ is updated at the sampling times $k=\widehat{k}_{j}, j \geq 1$.

(P.2) The local controller unit $\widehat{c}_{i}$ has direct access to the sensing unit $\widehat{s}_{i}$; consequently the local state $\widehat{x}_{i}\left[\widehat{k}_{j}\right]$ is assumed to be always available.

(P.3) The state information of neighboring stories obtained through the wireless communication unit $\widehat{w}_{i}$ has the form $\widehat{x}_{i^{\prime}}\left[k-\delta_{i^{\prime}}\left[\widehat{k}_{j}\right]\right]$, where $i^{\prime}=i \pm 1$ and the time delay satisfies $0 \leq \delta_{i^{\prime}}\left[\widehat{k}_{j}\right]<\widehat{r}$.

(P.4) For a given time interval $I$ with length $\widehat{\tau}$, the events $E_{i, i^{\prime}}(I, \widehat{\tau})=\left[\widehat{c}_{\mathrm{i}}\right.$ obtains the neighboring state $\widehat{x}_{i^{\prime}}$ in the time interval $I$ of length $\left.\widehat{\tau}\right]$ are independent random events with common probability

$$
\operatorname{Prob}\left[E_{i, i^{\prime}}(I, \widehat{\tau})\right]=p_{\hat{\tau}} .
$$

(P.5) If $I, I^{\prime}$ are non-overlapping time intervals with respective lengths $\widehat{\tau}$ and $\widehat{\tau}^{\prime}$, then $E_{i, i^{\prime}}(I, \widehat{\tau})$ and $E_{i, i^{\prime}}\left(I^{\prime}, \widehat{\tau}^{\prime}\right)$ are independent random events.

(P.6) Through the time interval $\Delta_{g}\left[\widehat{k}_{j}\right]$, the local control unit $\widehat{c}_{i}$ tries to collect the state information required to compute the control action. If this state information is 
successfully acquired, the flag variable $\phi_{i}\left[\widehat{k}_{j}\right]$ is set to 1 and the new control action $\widehat{u}_{i}\left[\widehat{k}_{j}\right]$ is computed; otherwise, the flag variable $\phi_{i}\left[\widehat{k}_{j}\right]$ and the control action $\widehat{u}_{i}\left[\widehat{k}_{j}\right]$ are both set to 0 .

(P.7) The control action computed at the sampling time $k=\widehat{k}_{j}$ is held through the time interval $\Delta_{h}\left[\widehat{k}_{j}\right]$.

According to the previous principles, the vector of control actions

$$
\widehat{u}[k]=\left[\widehat{u}_{1}[k], \ldots, \widehat{u}_{n}[k]\right]^{T}
$$

can be computed by setting the initial value

$$
\widehat{u}[0]=[0]_{n \times 1},
$$

and, for $k>0$, using the expression

$$
\widehat{u}[k]= \begin{cases}-F_{\phi}[k]\left(D_{\widehat{\mathrm{G}}_{\widehat{\tau}}} x[k]+L_{\widehat{\mathrm{G}}_{\widehat{\tau}}} x[k-\delta[k]]\right) & \text { if } \bmod (k, \widehat{r})=0, \\ \widehat{u}[k-1], & \text { otherwise }\end{cases}
$$

where $\bmod (k, \widehat{r})$ represents the integer remainder after division, $F_{\phi}[k]$ is the diagonal matrix

$$
F_{\phi}[k]=\left[\begin{array}{ccc}
\phi_{1}[k] & & \\
& \ddots & \\
& & \phi_{n}[k]
\end{array}\right]
$$

$D_{\widehat{\mathrm{G}}_{\hat{\tau}}}$ is the block-diagonal matrix

$$
D_{\widehat{\mathrm{G}}_{\hat{\tau}}}=\left[\begin{array}{llll}
{\left[\widehat{G}_{\widehat{\tau}}\right]_{1,1}} & & \\
& \ddots & \\
& & {\left[\widehat{G}_{\widehat{\tau}}\right]_{n, n}}
\end{array}\right]
$$

formed by the diagonal blocks of $\widehat{G}_{\hat{\tau}}$

$$
\left[\widehat{G}_{\widehat{\tau}}\right]_{i, i}=\left[\left\{\widehat{g}_{\widehat{\tau}}\right\}_{i, 2 i-1},\left\{\widehat{g}_{\widehat{\tau}}\right\}_{i, 2 i}\right], \quad 1 \leq i \leq n,
$$

and the matrix

$$
L_{\widehat{\mathrm{G}}_{\hat{\tau}}}=\widehat{\mathrm{G}}_{\hat{\tau}}-D_{\widehat{\mathrm{G}}_{\hat{\tau}}}
$$


contains the out-of-diagonal blocks of the block-tridiagonal multioverlapping control matrix $\widehat{G}_{\hat{\tau}}$. The notation $x[k-\delta[k]]$ represents the delayed state

$$
x[k-\delta[k]]=\left[\begin{array}{c}
\widehat{x}_{1}\left[k-\delta_{1}[k]\right] \\
\vdots \\
\widehat{x}_{n}\left[k-\delta_{n}[k]\right]
\end{array}\right],
$$

where $\delta_{i}[k]$ is the delay in the local state $\widehat{x}_{i}$.

The multioverlapping control model can now be obtained by completing (4.14) and (4.15) with the state and output equations

$$
\begin{gathered}
x[k+1]=A_{\tau} x[k]+B_{\tau} \sigma(\widehat{u}[k])+E_{\tau} \omega_{\tau}[k], \\
y[k]=C_{y} x[k] .
\end{gathered}
$$

It should be noted that expressions (4.16) and (4.20) are only evaluated at the update-control times $\widehat{k}_{j}=j \hat{r}$. Moreover, according to (P.1), (P.4) and (P.6), the information-state flag variables $\phi_{i}\left[\widehat{k}_{j}\right]$ are independent random variables with Bernoulli distributions $\mathcal{B}(p)$. In particular $\phi_{1}\left[\widehat{k}_{j}\right]$ and $\phi_{n}\left[\widehat{k}_{j}\right]$ have a Bernoulli distribution $B\left(p_{\widehat{\tau}}\right)$, and $\phi_{i}\left[\widehat{k}_{j}\right]$ has distribution $B\left(p_{\widehat{\tau}}^{2}\right)$ for $1<i<n$. Finally, it should also be noted that the probability of gathering the neighboring state information $\widehat{x}_{i^{\prime}}$ by the controller unit $\widehat{c}_{i}$ in a time interval $I^{\prime}$ of length $2 \widehat{\tau}$ is

$$
\operatorname{Prob}\left[E_{i, i^{\prime}}\left(I^{\prime}, 2 \widehat{\tau}\right)\right]=2 p_{\widehat{\tau}}-p_{\widehat{\tau}}^{2}
$$

This formula can be easily obtained by writing the time interval $I^{\prime}$ as union of two nonoverlapping intervals $I^{\prime}=I_{1}^{\prime} \cup I_{2}^{\prime}$ of length $\widehat{\tau}$. According to (P.5), $E_{i, i^{\prime}}\left(I_{1}^{\prime}, \widehat{\tau}\right)$ and $E_{i, i^{\prime}}\left(I_{2}^{\prime}, \widehat{\tau}\right)$ are independent random events, and the probability of failing to acquire the state $\widehat{x}_{i^{\prime}}$ in the whole interval $I^{\prime}$ is $\left(1-p_{\widehat{\tau}}\right)^{2}$. Analogously, it can be shown that the corresponding probability for a time interval $I^{\prime \prime}$ of length $(1 / 2) \widehat{\tau}$ is

$$
\operatorname{Prob}\left[E_{i, i^{\prime}}\left(I^{\prime \prime}, \frac{1}{2} \widehat{\tau}\right)\right]=1-\sqrt{1-p_{\hat{\tau}}}
$$

\section{Numerical Simulations}

In this section, the behavior of discrete-time multioverlapping LQR controllers is investigated through numerical simulations of the seismic response of a 20-story building. The parameter values for this particular building are collected in Table 1 and are similar to those used in [5]. The damping matrix has been computed as a Rayleigh damping matrix by setting a $5 \%$ of damping ratio for the 1 st and 18 th natural frequencies. The actuation system $a_{i}$ implemented between the $(i-1)$ th and $i$ th stories (see Figure 2 ) is assumed to be formed by a number of identical actuation devices that work coordinately as a single device. The force saturation level of a single actuation device has been taken as $1.2 \times 10^{6} \mathrm{~N}$. The total number of actuation devices and the maximum actuation force for the actuation systems $a_{i}, 1 \leq i \leq n$, is also presented in Table 1. 
Table 1: Particular parameter values for the 20-story building.

\begin{tabular}{lcccccc}
\hline & \multicolumn{5}{c}{ Story } \\
& $1-5$ & $6-11$ & $12-14$ & $15-17$ & $18-19$ & 20 \\
\hline Mass $\left(\times 10^{6} \mathrm{Kg}\right)$ & 1.10 & 1.10 & 1.10 & 1.10 & 1.10 & 1.10 \\
Stiffness $\left(\times 10^{6} \mathrm{~N} / \mathrm{m}\right)$ & 8.62 & 5.54 & 4.54 & 2.91 & 2.56 & 1.72 \\
Number of actuation devices & 4 & 2 & 2 & 1 & 1 & 1 \\
Max. actuation force $\left(\times 10^{6} \mathrm{~N}\right)$ & 4.8 & 2.4 & 2.4 & 1.2 & 1.2 & 1.2 \\
Natural damping & $5 \%$ & & & & & \\
\hline
\end{tabular}

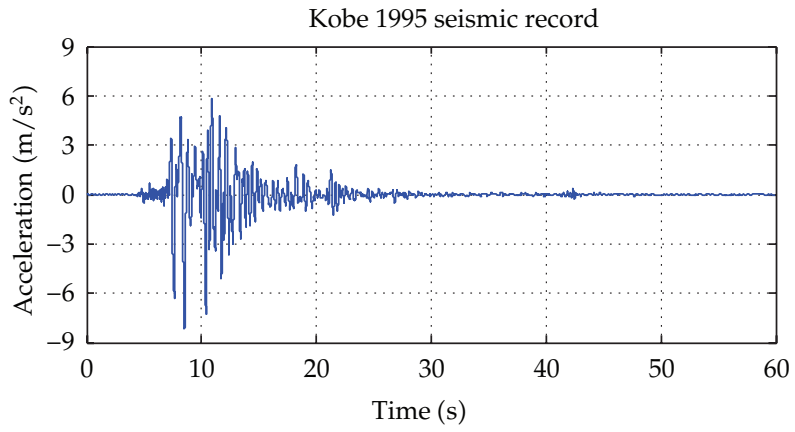

Figure 6: Full scale Kobe 1995 North-South seismic record.

For this 20-story building, three different discrete-time LQR controllers are designed: a centralized controller $G_{\tau}$, which has been obtained using the basic sampling time $\tau=10^{-3} \mathrm{~s}$; and two multioverlapping controllers $\widehat{G}_{\widehat{\tau}}$ and $\widehat{G}_{\hat{\tau}^{\prime}}$, computed with sampling times $\widehat{\tau}=40 \tau$, and $\widehat{\tau}^{\prime}=20 \tau$, respectively. The particular values of the weighting matrices in (3.5) used to design the centralized controller are $Q=I_{40}$ and $R=10^{-17.5} \times I_{20}$. For the multioverlapping controllers, the weighting matrices in (3.13) used to compute the local expanded LQR controllers $\widetilde{G}_{\tilde{\tau}}^{(i)}$ and $\widetilde{G}_{\tilde{\tau}^{\prime}}^{(i)}, 1 \leq i<20$, have been taken as $Q^{(i)}=I_{4}$ and $R^{(i)}=10^{-17.5} \times I_{2}$, for $1 \leq i<20$.

In the numerical simulations, the maximum absolute interstory drifts have been computed for different control configurations. The basic building model given in (4.1) with sampling time $\tau=10^{-3} \mathrm{~s}$ has been used to compute the uncontrolled seismic response. The controlled response corresponding to the centralized controller $G_{\tau}$ has been obtained with the centralized control model presented in (4.3). Finally, the multioverlapping control model defined in (4.14), (4.15), and (4.21) has been used to compute the controlled response for the multioverlapping controllers $\widehat{G}_{\hat{\tau}}$ and $\widehat{G}_{\hat{\tau}^{\prime}}$. In all the cases, the full scale 1995 Kobe North-South seismic record has been taken as ground acceleration (see Figure 6). This seismic record, obtained at the Kobe Japanese Meteorological Agency station during the Hyogoken-Nanbu earthquake of January 17, 1995, is a near-field record that presents large acceleration peaks which are extremely destructive to tall structures $[18,19]$. In the multioverlapping control model, the reference value of $p_{\widehat{\tau}}=0.95$ has been set for the probability of obtaining state information from neighboring stories in a time interval of length $\widehat{\tau}=40 \mathrm{~ms}$. According to (4.23), for the multioverlapping controller $\widehat{G}_{\hat{\tau}^{\prime}}$ with control sampling time $\widehat{\tau}^{\prime}=20 \mathrm{~ms}$, the probability of successfully gathering state information from neighboring stories can be taken as $p_{\widehat{\tau}^{\prime}}=0.78$. 


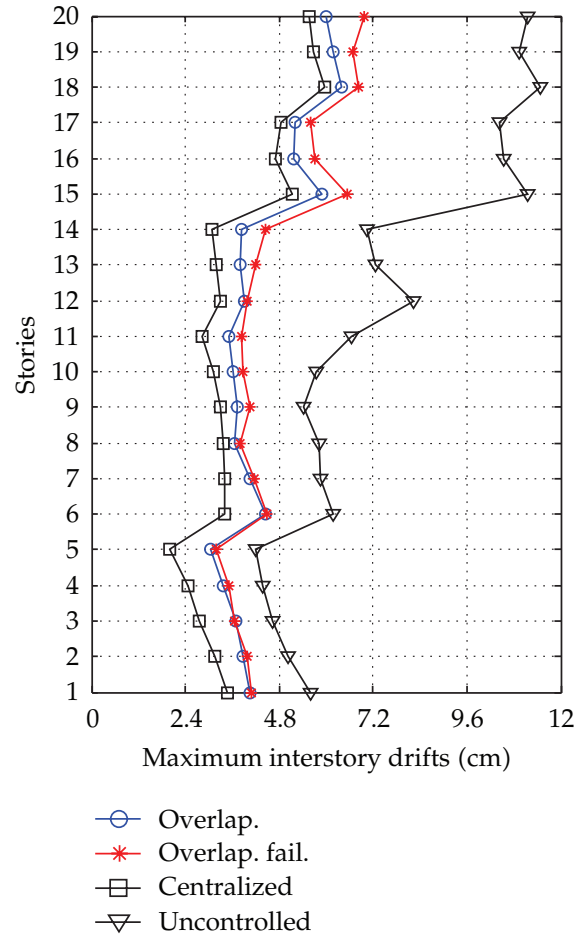

(a) Controller sampling time $40 \mathrm{~ms}$

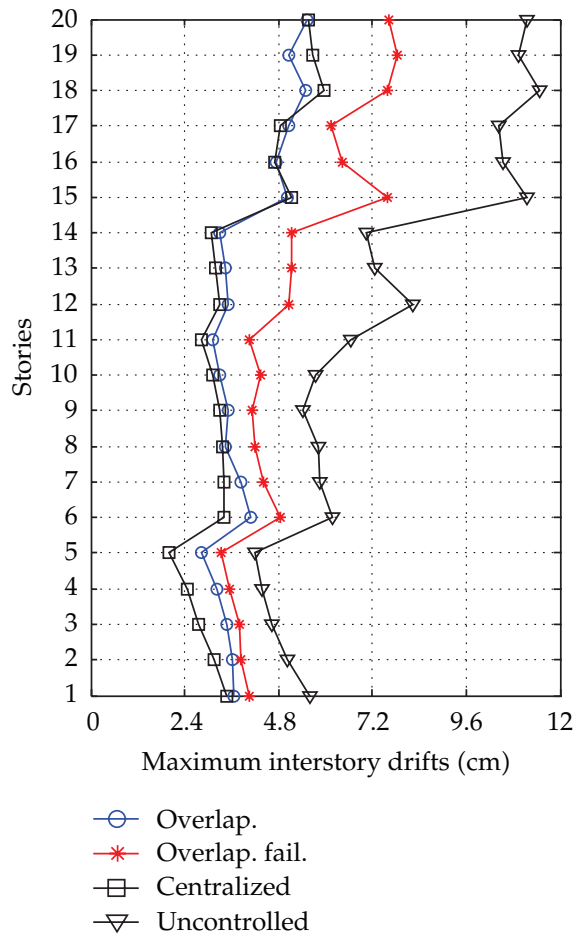

(b) Controller sampling time $20 \mathrm{~ms}$

Figure 7: Maximum absolute interstory drifts for the 1995 Kobe North-South seismic record. Simulations with maximum state delay.

In Figure 7(a), the red line with asterisks (Overlap. fail in the legend) presents the maximum absolute interstory drifts corresponding to the multioverlapping controller $\widehat{G}_{\hat{\tau}}$ with controller sampling time $\widehat{\tau}=40 \mathrm{~ms}$, probability of successful communication $p_{\hat{\tau}}=0.95$, and state delay $\delta=39 \mathrm{~ms}$. The blue line with circles (Overlap. in the legend), displays the values obtained with no communication failures, that is, with $p_{\hat{\tau}}=1$. The interstory drifts peak values corresponding to the multioverlapping controller $\widehat{G}_{\hat{\tau}^{\prime}}$ with controller sampling time $\widehat{\tau}^{\prime}=20 \mathrm{~ms}$, and state delay $\delta=19 \mathrm{~ms}$ are presented in Figure $7(\mathrm{~b})$. Here, the red line with asterisks corresponds to the probability $p_{\hat{\tau}^{\prime}}=0.78$, and the blue line with circles presents again the results for $p_{\hat{\tau}^{\prime}}=1$. In both cases, the graphics corresponding to the uncontrolled response (black line with triangles), and the controlled response for the centralized controller $G_{\tau}$, with controller sampling time $\tau=1 \mathrm{~ms}$, with no communication failures nor delays (black line with squares) have been included as reference. In Figure 8, the red line with asterisks (Over. delay in the legend) displays the maximum absolute interstory drifts corresponding to the multioverlapping controller $\widehat{G}_{\widehat{\tau}}$ with $\widehat{\tau}=40 \mathrm{~ms}, p_{\widehat{\tau}}=0.95$, and state delay $\delta=39 \mathrm{~ms}$, while the green line with circles (Over. no delay in the legend) presents the response obtained with null state delay.

The graphics in Figure 7(a) show the excellent performance of the proposed multioverlapping controller for controller sampling times $\widehat{\tau}$ compatible with moderate values of communication latency and also compatible with moderate rates of communication failures. Moreover, the graphics in Figure $7(b)$ clearly illustrate the trade-off between the controller 


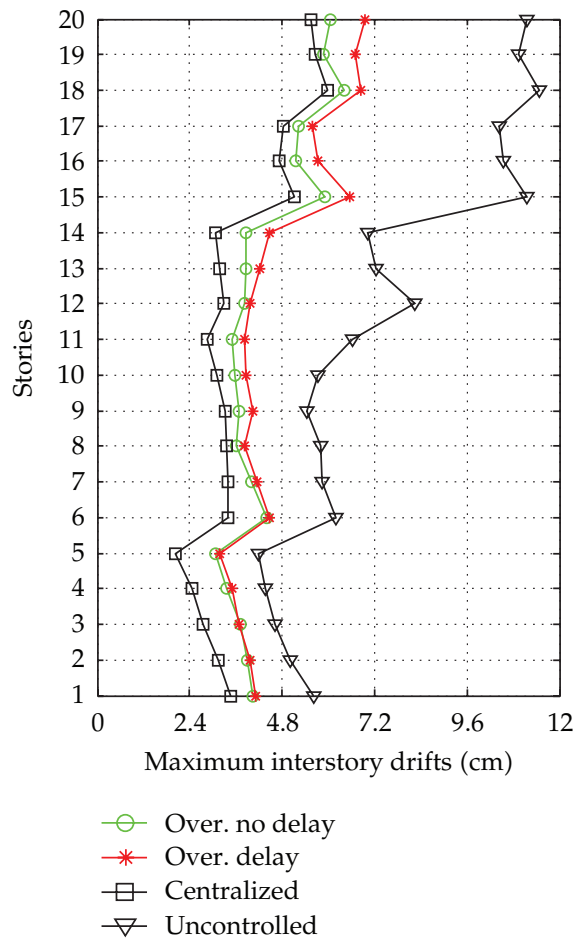

Figure 8: Maximum absolute interstory drifts for maximum and minimum state delays (controller sampling time $40 \mathrm{~ms})$.

sampling time $\widehat{\tau}$ and the probability of successful communication $p_{\hat{\tau}}$. Certainly, taking a smaller sampling time $\widehat{\tau}$ allows a more accurate implementation of the control actions; however, this also implies a reduction of the probability $p_{\hat{\tau}}$ which, in the end, may result in an overall loss of performance.

Finally, the graphics in Figure 8 show the moderate influence of the state delay $\delta$ in the multioverlapping controller performance for reasonable values of the controller sampling time $\widehat{\tau}$.

Remark 5.1. It is worth to be mentioned that the behavior of the ideal discrete-time centralized controller $G_{\tau}$ is very similar to the behavior exhibited by an ideal continuous-time centralized LQR controller. As mentioned in Remark 3.3, full state information is required by centralized controllers and this fact makes them unsuitable for SVC of large buildings with wireless communication systems. A detailed discussion of this point can be found in [5].

Remark 5.2. The proposed semidecentralized controllers can operate using only state information from neighboring stories. This fact makes it possible for them to successfully collect the required state information in a relatively small time interval. As a side effect, state delays are also small and have no significant impact on the controller performance.

Remark 5.3. Force saturation is an important issue in SVC. For large seismic excitations, the required control actions frequently exceed the force capacity of the actuation devices. Consequently, force actuation constraints should be considered when studying the controllers 
behavior. All the numerical simulations of the controlled responses presented in this paper have been conducted using the force saturation values displayed in Table 1.

\section{Conclusions and Future Directions}

In this paper, a computationally effective strategy has been used to design discrete-time state-feedback multioverlapping LQR controllers for seismic protection of tall buildings. This strategy, based on a sequential application of the Inclusion Principle, produces a blocktridiagonal control gain matrix that allows computing the corresponding control actions using only state information from neighboring stories. Due to this particular information exchange configuration of the multioverlapping controllers, the transmission range and the control sampling frequency in wireless implementations of the communication system can be dramatically improved. To investigate the behavior of the proposed semidecentralized multioverlapping controllers, a proper simulation model has been designed, which allows including semiactive actuation devices with limited force capacity, control sampling times consistent with the communication latency, time-delayed state information, and communication failures. To assess the performance of the proposed multioverlapping controllers, numerical simulations of the seismic response for a 20-story building model have been conducted with positive results.

For clarity and simplicity, the controllers presented in this paper have been designed following an LQR approach. In future works, further research effort should be addressed at exploring the effectiveness of the proposed control design strategy in more complex scenarios, which can involve issues of practical interest such as structural information constraints [20], actuator saturation [21], actuation and sensor failures [22], and limited frequency domain [23]. Other natural extensions of the present work should include a deeper treatment of some important practical aspects related to the communication system such as missing measurements [24-28], stochastic uncertainties [29], and stochastic nonlinearities [30-33].

\section{Acknowledgments}

This work has been partially supported by the Spanish Ministry of Economy and Competitiveness through the Grant DPI2011-25567-C02 and by the Norwegian Center of Offshore Wind Energy (NORCOWE) under Grant 193821/S60 from the Research Council of Norway $(\mathrm{RCN})$.

\section{References}

[1] B. F. Spencer and S. Nagarajaiah, "State of the art of structural control," Journal of Structural Engineering, vol. 129, no. 7, pp. 845-856, 2003.

[2] R. A. Swartz and J. P. Lynch, "Strategic network utilization in a wireless structural control system for seismically excited structures," Journal of Structural Engineering, vol. 135, no. 5, pp. 597-608, 2009.

[3] H. Li and L. Huo, "Advances in structural control in civil engineering in China," Mathematical Problems in Engineering, vol. 2010, Article ID 936081, 23 pages, 2010.

[4] Y. Wang and J. Lynch, "Wireless sensing and decentralized control for civil structures: theory and implementation," Tech. Rep. 167, J. A. Blume Engineering Center, Department of Civil and Environmental Engineering, Stanford University, Stanford, Calif, USA, 2007.

[5] Y. Wang, J. P. Lynch, and K. H. Law, “Decentralized $H_{\infty}$ controller design for large-scale civil structures," Earthquake Engineering and Structural Dynamics, vol. 38, no. 3, pp. 377-401, 2009. 
[6] J. Lunze, Feedback Control of Large-Scale Systems, Prentice Hall, Upper Saddle River, NJ, USA, 1992.

[7] D. D. Šiljak, Decentralized Control of Complex Systems, vol. 184 of Mathematics in Science and Engineering, Academic Press, Boston, Mass, USA, 1991.

[8] L. Bakule, J. Rodellar, and J. M. Rossell, "Generalized selection of complementary matrices in the inclusion principle," IEEE Transactions on Automatic Control, vol. 45, no. 6, pp. 1237-1243, 2000.

[9] L. Bakule, J. Rodellar, and J. M. Rossell, "Structure of expansion-contraction matrices in the inclusion principle for dynamic systems," SIAM Journal on Matrix Analysis and Applications, vol. 21, no. 4, pp. 1136-1155, 2000.

[10] A. I. Zečević and D. D. Šiljak, "A decomposition-based control strategy for large, sparse dynamic systems," Mathematical Problems in Engineering, no. 1, pp. 33-48, 2005.

[11] X.-B. Chen and S. S. Stanković, "Decomposition and decentralized control of systems with multioverlapping structure," Automatica, vol. 41, no. 10, pp. 1765-1772, 2005.

[12] A. I. Zečević and D. D. Šiljak, "Control of Complex Systems," Structural Constraints and Uncertainty, Springer, 2010.

[13] L. Bakule, F. Paulet-Crainiceanu, J. Rodellar, and J. M. Rossell, "Overlapping reliable control for a cable-stayed bridge benchmark," IEEE Transactions on Control Systems Technology, vol. 13, no. 4, pp. 663-669, 2005.

[14] J. M. Rossell, F. Palacios-Quiñonero, and J. Rodellar, "Semi-decentralized output feedback $H_{\infty}$ control strategy for large building structures," in Proceedings of the 5th World Conference on Structural Control and Monitoring, Tokyo, Japan, 2010.

[15] F. Palacios-Quiñonero, J. M. Rossell, and H. R. Karimi, "Semi-decentralized strategies in structural vibration control," Modeling Identification and Control, vol. 32, no. 2, pp. 57-77, 2011.

[16] F. Palacios-Quiñonero, J. Rodellar, and J. M. Rossell, "Sequential design of multi-overlapping controllers for longitudinal multi-overlapping systems," Applied Mathematics and Computation, vol. 217, no. 3, pp. 1170-1183, 2010.

[17] A. K. Chopra, Dynamics of Structures: Theory and Applications to Earthquake Engineering, Prentice Hall, Upper Saddle River, NJ, USA, 3rd edition, 2007.

[18] Y. Ohtori, R. E. Christenson, B. F. Spencer, and S. J. Dyke, "Benchmark control problems for seismically excited nonlinear buildings," Journal of Engineering Mechanics, vol. 130, no. 4, pp. 366-385, 2004.

[19] C. T. Huang and S. S. Chen, "Near-field characteristics and engineering implications of the 1999 ChiChi earthquake," Earthquake Engineering and Engineering Sysmology, vol. 2, no. 1, pp. 23-41, 2000.

[20] J. Rubió-Massegú, F. Palacios-Quiñonero, and J. M. Rossell, “Decentralized static output-feedback $H_{\infty}$ controller design for buildings under seismic excitation," Earthquake Engineering and Structural Dynamics, vol. 41, no. 7, pp. 1199-1205, 2012.

[21] H. Du and J. Lam, "Energy-to-peak performance controller design for building via static output feedback under consideration of actuator saturation," Computers $\mathcal{E}$ Structures, vol. 84, no. 31-32, pp. 2277-2290, 2006.

[22] W. Zhang, Y. Cheng, and H. Gao, "Energy-to-peak control for seismic-excited buildings with actuator faults and parameter uncertainties," Journal of Sound and Vibration, vol. 330, no. 4, pp. 581-602, 2011.

[23] Y. Chen, W. Zhan, and H. Gao, "Finite frequency $H_{\infty}$ control for building under earthquake excitation," Mechatronics, vol. 20, no. 1, pp. 128-142, 2010.

[24] H. Dong, Z. Wang, and H. Gao, " $H_{\infty}$ fuzzy control for systems with repeated scalar nonlinearities and random packet losses," IEEE Transactions on Fuzzy Systems, vol. 17, no. 2, pp. 440-450, 2009.

[25] B. Shen, Z. Wang, and Y. S. Hung, "Distributed $H_{\infty}$ consensus filtering in sensor networks with multiple missing measurements: the finite-horizon case," Automatica, vol. 46, no. 10, pp. 1682-1688, 2010.

[26] H. Dong, Z. Wang, and H. Gao, "Distributed filtering for a class of time-varying systems over sensor networks with quantization errors and successive packet dropouts," IEEE Transactions on Signal Processing, vol. 60, no. 6, pp. 3164-3173, 2012.

[27] Z. Wang, B. Shen, H. Shu, and G. Wei, "Quantized $H_{\infty}$ control for nonlinear stochastic time-delay systems with missing measurements," IEEE Transactions on Automatic Control, vol. 57, no. 6, pp. 14311444, 2012.

[28] Z. Wang, B. Shen, and X. Liu, " $H_{\infty}$ filtering with randomly occurring sensor saturations and missing measurements," Automatica, vol. 48, no. 3, pp. 556-562, 2012.

[29] J. Hu, Z. Wang, H. Gao, and L. K. Stergioulas, "Robust sliding mode control for discrete stochastic systems with mixed time delays, randomly occurring uncertainties, and randomly occurring nonlinearities," IEEE Transactions on Industrial Electronics, vol. 59, no. 7, pp. 3008-3015, 2012. 
[30] J. Hu, Z. Wang, Y. Niu, and L. K. Stergioulas, " $H_{\infty}$ sliding mode observer design for a class of nonlinear discrete time-delay systems: a delay-fractioning approach," International Journal of Robust and Nonlinear Control, vol. 22, no. 16, pp. 1806-1826, 2012.

[31] J. Hu, Z. Wang, and H. Gao, "A delay fractioning approach to robust sliding mode control for discretetime stochastic systems with randomly occurring non-linearities," IMA Journal of Mathematical Control and Information, vol. 28, no. 3, pp. 345-363, 2011.

[32] J. Hu, Z. Wang, H. Gao, and L. K. Stergioulas, "Robust $H_{\infty}$ sliding mode control for discrete timedelay systems with stochastic nonlinearities," Journal of the Franklin Institute, vol. 349, no. 4, pp. 1459 1479, 2012.

[33] J. Hu, Z. Wang, H. Gao, and L. K. Stergioulas, "Probability-guaranteed $H_{\infty}$ finite-horizon filtering for a class of nonlinear time-varying systems with sensor saturations," Systems $\mathcal{E}$ Control Letters, vol. 61, no. 4, pp. 477-484, 2012. 


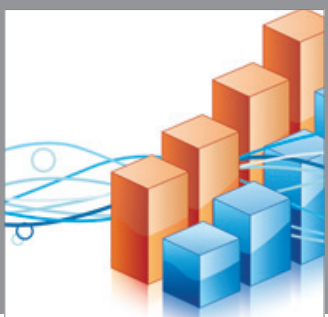

Advances in

Operations Research

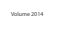

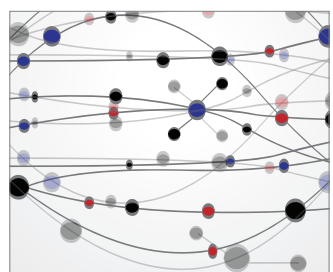

\section{The Scientific} World Journal
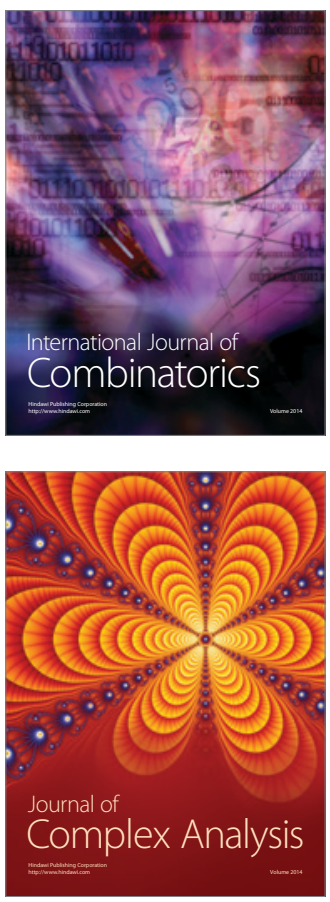

International Journal of

Mathematics and

Mathematical

Sciences
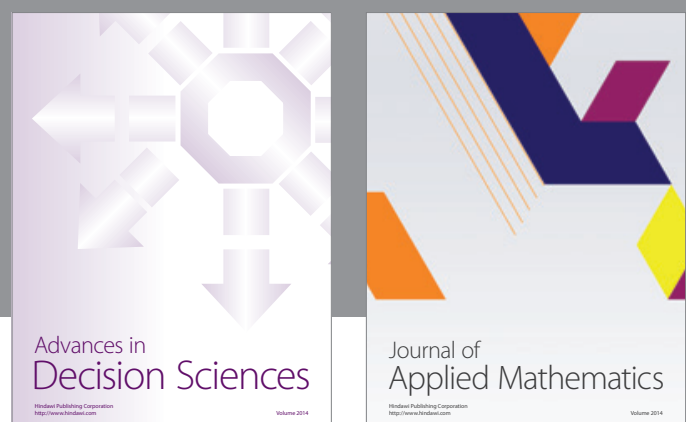

Journal of

Applied Mathematics
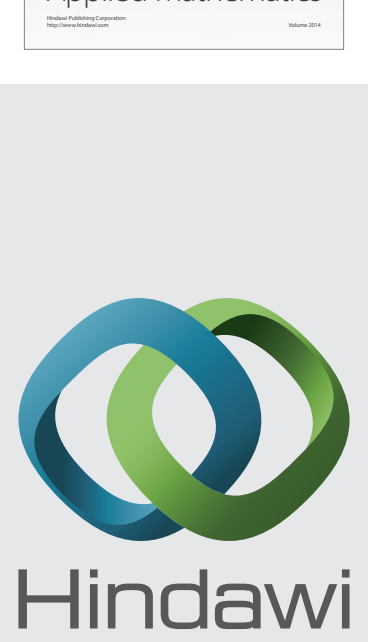

Submit your manuscripts at http://www.hindawi.com
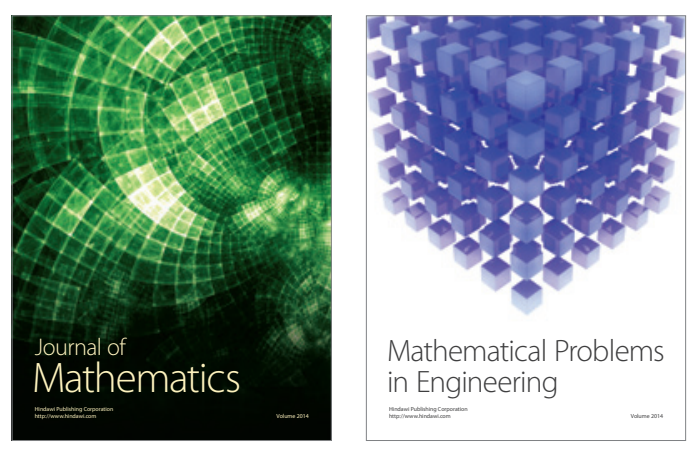

Mathematical Problems in Engineering
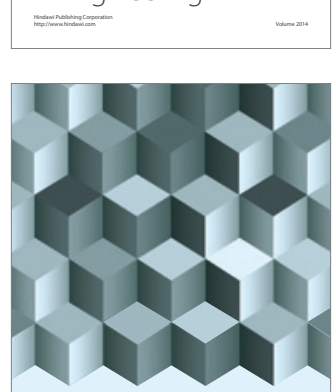

Journal of

Function Spaces
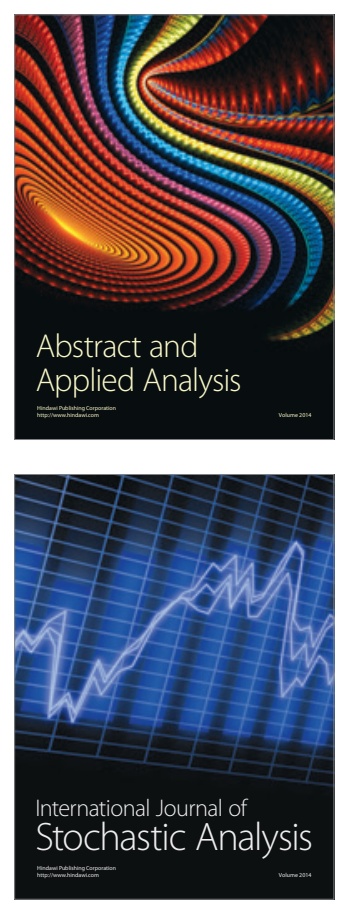

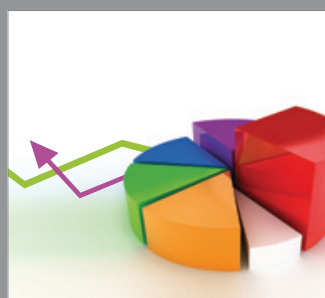

ournal of

Probability and Statistics

Promensencen
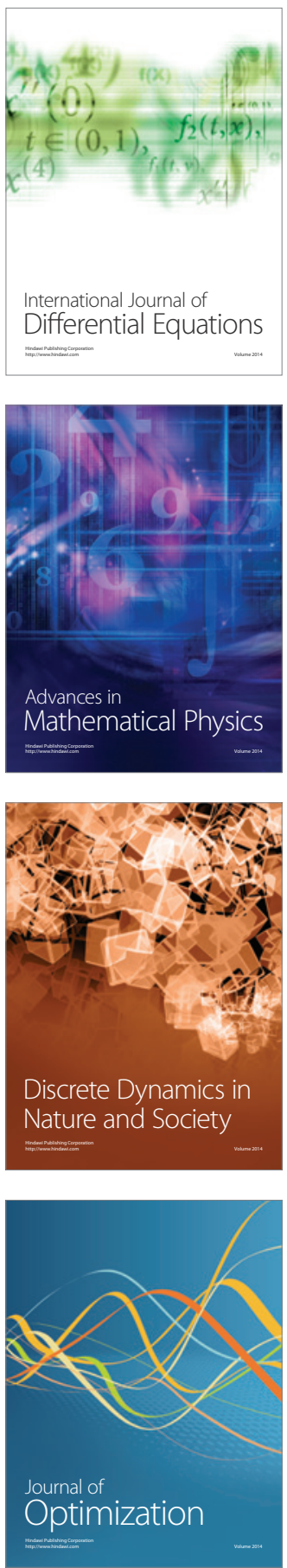